MATHEMATICS OF COMPUTATION

Volume 80, Number 274, April 2011, Pages 995-1009

S $0025-5718(2010) 02426-3$

Article electronically published on September 24, 2010

\title{
HIGH PRECISION COMPUTATION OF RIEMANN'S ZETA FUNCTION BY THE RIEMANN-SIEGEL FORMULA, I
}

\author{
J. ARIAS DE REYNA
}

\begin{abstract}
We present rigorous and sharp bounds for the terms and remainder in the Riemann-Siegel formula (for a general argument, not necessarily on the critical line). This allows for the computation of $\zeta(s)$ and $Z(t)$ to high precision. We also derive the Riemann-Siegel formula in a new and more direct way.
\end{abstract}

\section{INTRODUCTION}

Riemann only published one paper [7] about Number Theory. However, in the second edition of Riemann's Collected Works [8], published in 1892, the editor H. Weber included an excerpt of a draft of a letter from Riemann to Weierstrass, where Riemann speaks about a new expansion of the function $\Xi(t)$, not contained in 7. Some decades later Distel (the librarian of the Göttinger University) found in Riemann's Nachlass some sheets containing this expansion. In spite of this, it was not before 1932 that Siegel 9] published his paper about Riemann's results concerning the zeta function contained in Riemann's Nachlass.

Most probably, this expansion was used by Riemann to locate the first few nontrivial zeros of the zeta function. Starting with Titchmarsh and Comrie 11 and ending with Gourdon [6] this expansion has made it possible to show that the first $10^{13}$ non-trivial zeros of the zeta function are simple and are situated on the critical line.

Siegel also rescued an integral expression for the zeta function given by Riemann and found by Bessel-Hagen in Riemann's Nachlass:

$$
\zeta(s)=\mathcal{R}(s)+\chi(s) \overline{\mathcal{R}}(1-s) \quad \text { with } \quad \chi(s)=\pi^{s-1 / 2} \frac{\Gamma\left(\frac{1-s}{2}\right)}{\Gamma\left(\frac{s}{2}\right)},
$$

where

$$
\mathcal{R}(s):=\int_{0 \swarrow 1} \frac{x^{-s} e^{\pi i x^{2}}}{e^{\pi i x}-e^{-\pi i x}} d x \quad \text { and } \quad \overline{\mathcal{R}}(s):=\overline{\mathcal{R}(\bar{s})} .
$$

Gabcke 4, in 1979, used this integral expression to derive in a new way the asymptotic expansion of the zeta function given by Riemann (now known as the Riemann-Siegel (RS) formula). He also gave exact bounds of the first ten remainder terms for the critical line $\left(\sigma=\frac{1}{2}\right)$ allowing for precise computations and numerical verification of the Riemann Hypothesis $(\mathrm{RH})$.

Received by the editor December 3, 2009 and, in revised form, February 25, 2010. 2010 Mathematics Subject Classification. Primary 11M06, 11Y35; Secondary 65E05.

The author was supported by grant MTM2009-08934.

(c)2010 American Mathematical Society Reverts to public domain 28 years from publication 
Berry 2, in 1995, also gave precise bounds for the terms and remainder of the RS formula, but his arguments are not quite rigorous and are restricted to the critical line.

From (1.1) it is easily seen that

$$
Z(t)=2 \operatorname{Re}\left\{e^{i \vartheta(t)} \mathcal{R}\left(\frac{1}{2}+i t\right)\right\} \quad(t \in \mathbf{R}),
$$

where $\vartheta(t)$ is the phase of the zeta function $\zeta\left(\frac{1}{2}+i t\right)=Z(t) e^{-i \vartheta(t)}$ with $\vartheta(0)=0$. So, it is clear that in order to compute $\zeta(s)$ or $Z(t)$ it is sufficient to compute $\mathcal{R}(s)$. We may also restrict the computation of $\mathcal{R}(s)$ to $t=\operatorname{Im} s>0$. Our main goal will be to obtain an asymptotic expansion of $\mathcal{R}(s)$ for $t \rightarrow \infty$. (The behavior of $\mathcal{R}(s)$ for $t<0$ is very different from that for $t>0$.) The idea of deriving the Riemann-Siegel expansion from the integral for $\mathcal{R}(s)$ goes back to Gabcke.

We will obtain the expansion in a new way and also give new formulas of the terms of the expansion. In spite of the fact that Gabcke obtains the expansion for $Z(t)$ and we for $\mathcal{R}(s)$ our deduction is different although similar to that of Gabcke.

We will also present sharp and rigorous bounds of the terms and the remainder of the expansion. These results have allowed us to compose an algorithm to compute $\zeta(s)$ and $Z(t)$ to high precision for $t$ not too small. This algorithm has been implemented in Python using the Mpmath library for multiprecision floating-point arithmetic. The cpu time of this program is comparable to that of the proprietary software Mathematica. The Python program is explained in [1.

The computation of $\mathcal{R}(s)$ has also been treated in Galway [5] by applying quadrature techniques to the integral in (3.2), but precise error bounds for his scheme were not given.

In [1], we will present a complete analysis of the error committed when applying the Riemann-Siegel formula (for $t$ not too small).

\section{Summary of the main Results}

For $s=\sigma+i t$ with $t>0$, and an integer $K \geq 0$ we have

$$
\mathcal{R}(s)=\sum_{n=1}^{N} \frac{1}{n^{s}}+(-1)^{N-1} U a^{-\sigma}\left\{\sum_{k=0}^{K} \frac{C_{k}(p)}{a^{k}}+R S_{K}\right\},
$$

where

$$
U:=\exp \left\{-i\left(\frac{t}{2} \log \frac{t}{2 \pi}-\frac{t}{2}-\frac{\pi}{8}\right)\right\}
$$

and

$$
a:=\sqrt{\frac{t}{2 \pi}}, \quad N:=\lfloor a\rfloor, \quad p:=1-2(a-N)
$$

(for $R S_{K}$, see (3.8) and (3.10)).

The coefficients are given by

$$
C_{k}(p)=\frac{1}{\pi^{2 k}} \sum_{j=0}^{\lfloor 3 k / 2\rfloor}\left(\frac{\pi}{2 i}\right)^{j} d_{j}^{(k)} F^{(3 k-2 j)}(p),
$$

where $F$ is the entire function

$$
F(z)=\frac{e^{\pi i\left(\frac{z^{2}}{2}+\frac{3}{8}\right)}-i \sqrt{2} \cos \frac{\pi}{2} z}{2 \cos \pi z}=\sum_{n=0}^{\infty} c_{2 n} z^{2 n},
$$


where (the $E_{n}$ being the usual Euler numbers: $E_{0}=1, E_{2}=-1, E_{4}=5, E_{6}=-61$, ...)

$$
\begin{aligned}
c_{2 n}=-\frac{i}{\sqrt{2}}\left(\frac{\pi}{2}\right)^{2 n} \sum_{k=0}^{n} \frac{(-1)^{k}}{(2 k) !} 2^{2 n-2 k} & \frac{(-1)^{n-k} E_{2 n-2 k}}{(2 n-2 k) !} \\
& +e^{3 \pi i / 8} \sum_{j=0}^{n}(-1)^{j} \frac{E_{2 j}}{(2 j) !} \frac{i^{n-j} \pi^{n+j}}{(n-j) ! 2^{n-j+1}} .
\end{aligned}
$$

The coefficients $d_{j}^{(k)}$ appearing in (2.4) only depend on $\sigma$ and not on $t$ and can be computed by means of the following recurrences:

$$
\begin{aligned}
& d_{0}^{(0)}=1, \quad d_{j}^{(0)}=0 \quad \text { for } j \neq 0, \\
& d_{j}^{(k)}=0 \quad \text { for } \quad j<0 \quad \text { and for } \quad j>3 k / 2, \\
& (6 k-4 j) d_{j}^{(k)}=\frac{1}{2} d_{j}^{(k-1)}+(1-2 \sigma) d_{j-1}^{(k-1)}-2(3 k-2 j)(3 k-2 j+1) d_{j-2}^{(k-1)}, \\
& d_{3 k / 2}^{(k)}=-\sum_{j=0}^{3 k / 2-1}(-1)^{3 k / 2-j} d_{j}^{(k)} \frac{(3 k-2 j) !}{(3 k / 2-j) !}, \quad 3 k \equiv 0 \quad(\bmod 2) .
\end{aligned}
$$

In Theorems 4.1 and 4.2 we give bounds for the terms and the remainder of the expansion (2.1). We will also derive some other bounds that are necessary for proving in 1 that our (Python) program computes $\mathcal{R}(s)$ with an error less than (almost) any prescribed positive quantity.

\section{Riemann-Siegel Formula}

We assume that $s=\sigma+i t$ with $\sigma$ and $t$ real and $t>0$, and put, as usual,

$$
a=\sqrt{\frac{t}{2 \pi}}, \quad N=\lfloor a\rfloor .
$$

By Cauchy's Theorem we have

$$
\mathcal{R}(s)=\sum_{n=1}^{N} \frac{1}{n^{s}}+\int_{N_{\swarrow} N+1} \frac{x^{-s} e^{\pi i x^{2}}}{e^{\pi i x}-e^{-\pi i x}} d x,
$$

where $N \swarrow N+1$ denotes the straight line having direction $e^{-3 \pi i / 4}$ and containing the point $a$ (if $a=N$ is an integer we modify the path by means of a small semicircle with center at $a$, and intersecting the real axis between $N$ and $N+1$ ).

Now we modify the integrand as follows:

$$
\begin{aligned}
& x^{-s} e^{\pi i x^{2}}=\exp \{\left.\pi i(x-a+a)^{2}-(\sigma+i t) \log (x-a+a)\right\} \\
&=\exp \left\{-(\sigma+i t) \log a+\pi i a^{2}\right\} \\
& \times \exp \left\{\pi i(x-a)^{2}+2 \pi i a(x-a)-(\sigma+i t) \log \left(1+\frac{x-a}{a}\right)\right\} .
\end{aligned}
$$


Hence

$$
\begin{aligned}
\mathcal{R}(s) & =\sum_{n=1}^{N} \frac{1}{n^{s}} \\
+ & \left(\frac{t}{2 \pi}\right)^{-\sigma / 2} \exp \left\{-i\left(\frac{t}{2} \log \frac{t}{2 \pi}-\frac{t}{2}\right)\right\} \int_{N_{\swarrow} N+1} \frac{e^{2 \pi i(x-a)^{2}}}{e^{\pi i x}-e^{-\pi i x}} \widetilde{g}(a, x-a) d x,
\end{aligned}
$$

where

Therefore

$$
\widetilde{g}(a, z)=\exp \left\{-(\sigma+i t) \log \left(1+\frac{z}{a}\right)+2 \pi i a z-\pi i z^{2}\right\} .
$$

$$
\mathcal{R}(s)=\sum_{n=1}^{N} \frac{1}{n^{s}}+(-1)^{N-1}\left(\frac{t}{2 \pi}\right)^{-\sigma / 2} U \cdot S,
$$

where

$$
\begin{aligned}
& U=\exp \left\{-i\left(\frac{t}{2} \log \frac{t}{2 \pi}-\frac{t}{2}-\frac{\pi}{8}\right)\right\}, \\
& S=\frac{(-1)^{N-1}}{2 i} e^{-i \pi / 8} \int_{N_{\swarrow} N+1} \frac{e^{2 \pi i(x-a)^{2}}}{\sin \pi x} \widetilde{g}(a, x-a) d x .
\end{aligned}
$$

We change variables in the integral, taking $x=\frac{i v}{2}+N+\frac{1}{2}$, and define

$$
\tau=\frac{1}{2 \sqrt{2 t}} \quad \text { and } \quad p=1-2(a-N)
$$

Then we get

$$
t=\frac{1}{8 \tau^{2}}, \quad a=\frac{1}{4 \sqrt{\pi} \tau}, \quad N+\frac{1}{2}-a=\frac{p}{2}
$$

and, since $v=2 i\left(N+\frac{1}{2}-x\right)$, the integration path $N \swarrow N+1$ is converted into $\nwarrow i p$. This is a line having direction $e^{3 \pi i / 4}$ and containing the point $i p$. The point $i p$ is always on the segment joining the points $i$ and $-i$. (When $i p=i$ the path is modified by means of a small semicircle with center at $i p$ and intersecting the imaginary axis between $i$ and $-i$.)

Since

$$
\begin{gathered}
x-a=i \frac{v}{2}+N+\frac{1}{2}-a=i \frac{v-i p}{2}, \\
2 \pi i(x-a)^{2}=-\frac{\pi i}{2}(v-i p)^{2}=-\frac{\pi i}{2} v^{2}-\pi p v+\frac{\pi i}{2} p^{2} \\
\text { and } \sin \pi x=\cos \pi\left(i \frac{v}{2}+N\right)=(-1)^{N} \cosh \frac{\pi}{2} v
\end{gathered}
$$

we get, changing a sign for the sense of the path of integration,

$$
S=\frac{e^{-i \pi / 8}}{4} \int_{\searrow i p} \frac{e^{-\frac{\pi i}{2}(v-i p)^{2}}}{\cosh \frac{\pi}{2} v} \widetilde{g}\left(\frac{1}{4 \sqrt{\pi} \tau}, i \frac{v-i p}{2}\right) d v .
$$

To simplify the notation we put

$$
g(\tau, z)=\widetilde{g}\left(\frac{1}{4 \sqrt{\pi} \tau}, i \frac{z}{2 \sqrt{\pi}}\right)
$$

so that

$$
S=\frac{e^{-\pi i / 8}}{4} \int_{\searrow i p} \frac{e^{-\frac{\pi i}{2}(v-i p)^{2}}}{\cosh \frac{\pi}{2} v} g(\tau, \sqrt{\pi}(v-i p)) d v
$$


with

$$
g(\tau, z)=\exp \left\{-\left(\sigma+\frac{i}{8 \tau^{2}}\right) \log (1+2 i \tau z)-\frac{z}{4 \tau}+i \frac{z^{2}}{4}\right\} .
$$

From the expansion

$$
g(\tau, z)=\sum_{k=0}^{\infty} P_{k}(z) \tau^{k}=\sum_{k=0}^{K} P_{k}(z) \tau^{k}+R g_{K}(\tau, z) \quad(2 \tau|z|<1),
$$

it follows that the $P_{k}(z)$ are polynomials in $z$ and $\sigma$.

For every $K \geq 0$ we can now write

$$
\begin{aligned}
S=\sum_{k=0}^{K} \frac{e^{-\pi i / 8}}{4} \tau^{k} \int_{\searrow i p} \frac{e^{-\frac{\pi i}{2}(v-i p)^{2}}}{\cosh \frac{\pi}{2} v} P_{k}(\sqrt{\pi}(v-i p)) d v \\
\quad+\frac{e^{-\pi i / 8}}{4} \int_{\searrow i p} \frac{e^{-\frac{\pi i}{2}(v-i p)^{2}}}{\cosh \frac{\pi}{2} v} R g_{K}(\tau, \sqrt{\pi}(v-i p)) d v .
\end{aligned}
$$

Since $\tau=1 /(4 \sqrt{\pi} a)$ we get

$$
S=\sum_{k=0}^{K} \frac{C_{k}(p)}{a^{k}}+R S_{K}
$$

where, by definition,

$$
\begin{aligned}
C_{k}(p) & :=\frac{1}{4}\left(\frac{1}{4 \sqrt{\pi}}\right)^{k} e^{-\pi i / 8} \int_{\searrow i p} \frac{e^{-\frac{\pi i}{2}(v-i p)^{2}}}{\cosh \frac{\pi}{2} v} P_{k}(\sqrt{\pi}(v-i p)) d v \\
R S_{K} & :=\frac{e^{-\pi i / 8}}{4} \int_{\searrow i p} \frac{e^{-\frac{\pi i}{2}(v-i p)^{2}}}{\cosh \frac{\pi}{2} v} R g_{K}(\tau, \sqrt{\pi}(v-i p)) d v
\end{aligned}
$$

Now we can state

Theorem 3.1 (Lehmer's form of the Riemann-Siegel formula). Given $s=\sigma+i t$ with $t>0$, and an integer $K \geq 0$ we have

$$
\mathcal{R}(s)=\sum_{n=1}^{N} \frac{1}{n^{s}}+(-1)^{N-1} U a^{-\sigma}\left\{\sum_{k=0}^{K} \frac{C_{k}(p)}{a^{k}}+R S_{K}\right\},
$$

where $a$ and $N$ are defined by (3.1), $U$ by (3.3), $p$ by (3.4), $C_{k}(p)$ and $R S_{K}$ by (3.9) and (3.10).

\section{A Sharp BOUND FOR THE ERROR IN THE RS FORMUlA}

Theorem 4.1. Given $s=\sigma+i t$ with $t>0$, the coefficients $C_{k}(p)$ in the RiemannSiegel formula (3.11) satisfy the following inequality:

$$
\frac{\left|C_{k}(p)\right|}{a^{k}} \leq c \frac{\Gamma(k / 2)}{(b a)^{k}} \quad(k \geq 1),
$$

where the coefficients $c=c(\sigma)$ and $b=b(\sigma)$ are defined by

$$
c(\sigma):=\left\{\begin{array}{ll}
9^{\sigma} /(\sqrt{2} \pi) & \text { if } \sigma>0, \\
2^{-\sigma} /(\sqrt{2} \pi) & \text { if } \sigma \leq 0,
\end{array} \quad b(\sigma):= \begin{cases}2 & \text { if } \sigma>0 \\
\sqrt{(3-2 \log 2) \pi} & \text { if } \sigma \leq 0\end{cases}\right.
$$


That is,

$$
\left|C_{k}(p)\right| \leq \frac{2^{\frac{1}{2}-\sigma} \Gamma(k / 2)}{2 \pi(h \pi)^{k / 2}} \quad \text { if } \sigma \leq 0 \quad \text { and } \quad\left|C_{k}(p)\right| \leq \frac{\sqrt{2}}{2 \pi} \frac{9^{\sigma} \Gamma(k / 2)}{2^{k}} \quad \text { if } \sigma>0,
$$

where $h=3-2 \log 2 \approx 1.61371$.

Proof. The function $g(\tau, z)$ is an analytic function of $\tau$ on a neighborhood of $\tau=0$. (The logarithm in the definition of $g(\tau, z)$ is the principal logarithm.) By Cauchy's Theorem the polynomials $P_{k}(z)$ in (3.7) are given by

$$
P_{k}(z)=\frac{1}{2 \pi i} \int_{C} \frac{g(\zeta, z)}{\zeta^{k+1}} d \zeta,
$$

where $C$ is a small circle with center at $\zeta=0$. We apply a change of variable in this integral by putting $i \zeta /(2 z)$ instead of $\zeta$ :

$$
P_{k}(z)=\frac{(-2 i z)^{k}}{2 \pi i} \int_{C} \frac{(1-\zeta)^{-\sigma}}{\zeta^{k}} e^{-i z^{2} f(\zeta) / 2} \frac{d \zeta}{\zeta},
$$

where

$$
f(\zeta)=-\frac{\log (1-\zeta)}{\zeta^{2}}-\frac{1}{\zeta}-\frac{1}{2}
$$

Substituting this in the definition of $C_{k}(p)$ and applying Fubini's Theorem (that this is allowed will be clear after we will have bounded the integrals) we get

$$
\begin{aligned}
& C_{k}(p) \\
& \quad=\frac{e^{-\pi i / 8}}{8 \pi i(2 i)^{k}} \int_{C} \frac{(1-\zeta)^{-\sigma}}{\zeta^{k}}\left\{\int_{\searrow i p} \frac{e^{-\frac{\pi i}{2}(v-i p)^{2}}}{\cosh \frac{\pi}{2} v}(v-i p)^{k} e^{-\frac{\pi i}{2}(v-i p)^{2} f(\zeta)} d v\right\} \frac{d \zeta}{\zeta},
\end{aligned}
$$

where $C$ is a circle with center $\zeta=0$ and radius $r<1$.

First we bound the absolute value of the integral with respect to $v$. We put $v=i p+\varepsilon x$, where $\varepsilon=e^{-\pi i / 4}$, so that $\varepsilon^{2}=-i$, and

$$
-\frac{\pi i}{2}(v-i p)^{2}=-\frac{\pi x^{2}}{2} .
$$

Writing $V(\zeta):=\operatorname{Re} f(\zeta)$, we have

$$
\left|\exp \left\{-\frac{\pi i}{2}(v-i p)^{2} f(\zeta)\right\}\right|=\exp \left(-\frac{\pi}{2} x^{2} V(\zeta)\right)
$$

so that for the inner integral,

$$
\left|\int_{\searrow i p} \cdots\right| \leq \int_{-\infty}^{+\infty} \frac{e^{-\pi x^{2} / 2}}{\left|\cosh \frac{\pi}{2}(i p+\varepsilon x)\right|}|x|^{k} e^{-\frac{\pi}{2} x^{2} V(\zeta)} d x .
$$

Since for $x, y \in \mathbf{R}$ we have $|\cosh (x+i y)|^{2}=\sinh ^{2} x+\cos ^{2} y$ it is easy to see that

$$
\left|\frac{x}{\cosh \frac{\pi}{2}(i p+\varepsilon x)}\right| \leq \frac{|x|}{\sinh \frac{\pi \sqrt{2}}{4}|x|} \leq \frac{4}{\pi \sqrt{2}} .
$$

$V(\zeta)$ is harmonic on the unit disc, and it can be shown (see footnote on page 1006) that $V\left(e^{i \theta}\right) \geq V(-1)=\frac{1}{2}-\log 2 \approx-0.193147$.

Hence, for $k \geq 1$,

$$
\left|\int_{\searrow i p} \ldots\right| \leq \frac{4}{\pi \sqrt{2}} \int_{-\infty}^{+\infty} e^{-\{1+V(\zeta)\} \frac{\pi x^{2}}{2}}|x|^{k-1} d x=\frac{2 \sqrt{2}}{\pi} \frac{(2 / \pi)^{k / 2} \Gamma(k / 2)}{(1+V(\zeta))^{k / 2}}
$$


and it follows that

$$
\begin{aligned}
\left|C_{k}(p)\right| & \leq \frac{\sqrt{2}}{4 \pi^{2}} \frac{\Gamma(k / 2)}{(2 \pi)^{k / 2}} \int_{C} \frac{1}{r^{k+1}}|1-\zeta|^{-\sigma}(1+V(\zeta))^{-k / 2}|d \zeta| \\
& =\frac{\sqrt{2}}{4 \pi^{2}} \frac{\Gamma(k / 2)}{(2 \pi)^{k / 2}} \int_{C}|1-\zeta|^{-\sigma} \frac{|d \zeta|}{r\left(r^{2}(1+V(\zeta))\right)^{k / 2}} .
\end{aligned}
$$

Consider first the case $\sigma \leq 0$. The radius of the circle $C$ can be any number in $(0,1)$. A good choice is to take the limit value $r \rightarrow 1$. For this choice $1+V\left(e^{i \theta}\right) \geq$ $h / 2$, where $h=2(1+V(-1))=3-2 \log 2 \approx 1.61371$. Therefore, for $k \geq 1$ and $\sigma \leq 0$ we get

$$
\left|C_{k}(p)\right| \leq \frac{2^{\frac{1}{2}-\sigma} \Gamma(k / 2)}{2 \pi(h \pi)^{k / 2}} .
$$

If $\sigma>0$ we cannot take $r=1$. Taking for example $r=8 / 9$ (see footnote on page 1006) we find that $r^{2}\left(1+V\left(r e^{i \theta}\right)\right) \geq 0.647961,\left|1-r e^{i \theta}\right| \geq 1 / 9$ and

$$
\left|C_{k}(p)\right| \leq \frac{\sqrt{2}}{2 \pi} \frac{9^{\sigma} \Gamma(k / 2)}{2^{k}} .
$$

It is easy to get various upper estimates of the same type: $C v^{\sigma} \Gamma(k / 2) b^{-k}$. Taking $v$ larger we can also take $b$ larger.

Theorem 4.2. Let $s=\sigma+i$ with $t>0$. Assume that $\sigma \geq 0$ or $K+\sigma \geq 2$. With the same notation as in Theorem 3.1 we have in (3.11),

$$
\left|R S_{K}\right| \leq c_{1} \frac{\Gamma((K+1) / 2)}{\left(b_{1} a\right)^{K+1}} \quad(K \geq 1),
$$

where $b_{1}=10 / 11=1 / 1.1$ and

$$
c_{1}:= \begin{cases}\frac{1}{7} 2^{3 \sigma / 2} & \text { for } \sigma \geq 0, \\ \frac{1}{2}\left(\frac{9}{10}\right)^{\lceil-\sigma\rceil} & \text { for } \sigma<0 .\end{cases}
$$

Proof. By (3.7) and Cauchy's Theorem we have

$$
R g_{K}(\tau, z)=\frac{1}{2 \pi i} \int_{C} \frac{g(\zeta, z)}{\zeta-\tau}\left(\frac{\tau}{\zeta}\right)^{K+1} d \zeta
$$

where the path of integration $C$ encircles the points 0 and $\tau$. We change variables by putting $i \zeta /(2 z)$ instead of $\zeta$. In this way we can show that

$$
R g_{K}(\tau, z)=\frac{(-2 i z \tau)^{K+1}}{2 \pi i} \int_{C} \frac{1}{(\zeta+2 i z \tau) \zeta^{K}}(1-\zeta)^{-\sigma} e^{-\frac{i z^{2}}{2} f(\zeta)} \frac{d \zeta}{\zeta},
$$

where now $C$ denotes a simple path that encircles the points 0 and $-2 i z \tau$ and is contained in the region obtained from $\mathbf{C}$ by making a cut along the real axis from 1 to $+\infty$. We will apply this for each point $z=v-i p$. So, this path cannot be the same for all values of $z$. Since $-2 i z \tau$, for $z=v-i p=\varepsilon x$, is on the line through 0 with direction $i \varepsilon=e^{\pi i / 4}$ we can always take as the path of integration the line $L$ through $1 / 2$ with direction $e^{\pi i / 4}$. For all $z=v-i p$ we have by Cauchy's Theorem,

$$
R g_{K}(\tau, z)=\frac{(-2 i z \tau)^{K+1}}{2 \pi i} \int_{L} \frac{1}{(\zeta+2 i z \tau) \zeta^{K}}(1-\zeta)^{-\sigma} e^{-\frac{i z^{2}}{2} f(\zeta)} \frac{d \zeta}{\zeta}
$$


We substitute this formula in (3.10) and apply Fubini's theorem to get

$$
\begin{aligned}
R S_{K}(\tau)= & \frac{(-2 i \tau \sqrt{\pi})^{K+1}}{8 \pi i} e^{-\pi i / 8} \\
& \times \int_{L}(1-\zeta)^{-\sigma} \frac{d \zeta}{\zeta^{K+1}} \int_{\searrow i p} \frac{e^{-\frac{\pi i}{2}(v-i p)^{2}}}{\cosh \frac{\pi}{2} v} \frac{(v-i p)^{K+1} e^{-\frac{\pi i}{2}(v-i p)^{2} f(\zeta)}}{(\zeta+2 i \sqrt{\pi}(v-i p) \tau)} d v .
\end{aligned}
$$

If $\zeta$ is on $L$ and $v=i p+\varepsilon x$, then $2 i \sqrt{\pi}(v-i p) \tau=2 i \sqrt{\pi} \varepsilon x \tau$ is on a line parallel to $L$ so that

$$
|\zeta+2 i \sqrt{\pi}(v-i p) \tau| \geq \frac{1}{2 \sqrt{2}}
$$

Then the interior integral can be bounded similarly as in (4.6), and we get

$$
\begin{aligned}
\left|R S_{K}(\tau)\right| \leq \frac{(2 \tau \sqrt{\pi})^{K+1}}{8 \pi} & 2 \sqrt{2} \int_{L} \frac{|1-\zeta|^{-\sigma}}{|\zeta|^{K+1}} \frac{2 \sqrt{2}}{\pi} \frac{(2 / \pi)^{(K+1) / 2} \Gamma((K+1) / 2)}{(1+V(\zeta))^{(K+1) / 2}}|d \zeta| \\
\leq & \frac{\left(8 \tau^{2}\right)^{(K+1) / 2}}{\pi^{2}} \Gamma\left(\frac{K+1}{2}\right) \int_{L} \frac{|1-\zeta|^{-\sigma}|d \zeta|}{\left\{|\zeta|^{2}(1+V(\zeta))\right\}^{(K+1) / 2}} .
\end{aligned}
$$

The line $L$ is given by the equation $\zeta=\frac{1}{2}+\frac{y}{2}(1+i)$ and, since $V(\zeta)=\operatorname{Re} f(\zeta)$, we arrive after some computation at

$$
\begin{array}{r}
1+V(\zeta)=\frac{1}{2}-\frac{2(1+y)}{(1+y)^{2}+y^{2}}-\frac{8\left(y+y^{2}\right)}{\left\{(1+y)^{2}+y^{2}\right\}^{2}}\left(\arctan (1-2 y)-\frac{\pi}{4}\right) \\
-\frac{2(1+2 y)}{\left\{(1+y)^{2}+y^{2}\right\}^{2}} \log \frac{(1-y)^{2}+y^{2}}{4} .
\end{array}
$$

This is always positive, has the limit $1 / 2$ when $y \rightarrow \infty$, and has an absolute maximum of 1.39959 at $y=0.396765$ and an absolute minimum 0.43068 at $y=$ 6.32884. So the integral in (4.9) converges for $K+\sigma>0$.

Assume first that $\sigma \geq 0$. Since $|1-\zeta| \geq 1 /(2 \sqrt{2})$ we have

$$
\left|R S_{K}(\tau)\right| \leq\left(8 \tau^{2}\right)^{(K+1) / 2} \frac{\Gamma((K+1) / 2)}{\pi^{2}}(2 \sqrt{2})^{\sigma} \int_{L} \frac{|d \zeta|}{\left\{(1+V(\zeta))|\zeta|^{2}\right\}^{(K+1) / 2}} .
$$

We now use the inequality $\|f\|_{p}^{p} \leq\|f\|_{1}\|f\|_{\infty}^{p-1}$. In this case,

$$
\int_{L} \frac{|d \zeta|}{\left\{(1+V(\zeta))|\zeta|^{2}\right\}} \approx 9.577048 \text { and } \max \frac{1}{\left\{(1+V(\zeta))|\zeta|^{2}\right\}} \approx 7.489341
$$

so that

$$
\left|R S_{K}(\tau)\right| \leq\left(8 \tau^{2}\right)^{(K+1) / 2} \frac{\Gamma((K+1) / 2)}{\pi^{2}}(2 \sqrt{2})^{\sigma} 9.577048(7.49)^{(K-1) / 2} .
$$

Observing that

we find that

$$
4 \tau=\frac{\sqrt{2}}{\sqrt{t}}=\frac{\sqrt{2}}{\sqrt{2 \pi}} \frac{\sqrt{2 \pi}}{\sqrt{t}}=\frac{1}{\sqrt{\pi}} \frac{1}{a},
$$

$$
\begin{aligned}
\left|R S_{K}(\tau)\right| & \leq \frac{9.578}{7.49 \pi^{2}} 2^{3 \sigma / 2} \Gamma((K+1) / 2)\left(\frac{\sqrt{7.49}}{\sqrt{2 \pi} a}\right)^{K+1} \\
& \leq \frac{1}{7} 2^{3 \sigma / 2} \Gamma((K+1) / 2)\left(\frac{1.1}{a}\right)^{K+1} .
\end{aligned}
$$


Now, for $\sigma<0$ let $m=\lceil-\sigma\rceil$ so that $-m \leq \sigma<1-m$. Since we assume $K+\sigma \geq 2$ we have $K>m+1$ and again by (4.9),

$$
\begin{aligned}
\left|R S_{K}(\tau)\right| \leq \frac{\left(8 \tau^{2}\right)^{(K+1) / 2}}{\pi^{2}} & \Gamma\left(\frac{K+1}{2}\right) \\
& \quad \times \int_{L}\left(\frac{|1-\zeta|^{2}}{\left\{|\zeta|^{2}(1+V(\zeta))\right\}}\right)^{m / 2} \frac{|1-\zeta|^{-\sigma-m}|d \zeta|}{\left\{|\zeta|^{2}(1+V(\zeta))\right\}^{(K-m+1) / 2}} .
\end{aligned}
$$

It can be shown that

$$
\frac{|1-\zeta|^{2}}{\left\{|\zeta|^{2}(1+V(\zeta))\right\}} \leq 5.78453 \quad(\zeta \in L)
$$

the left-hand side being maximal for $\zeta \approx 0.121696-i 0.378304$. Since $0 \leq \sigma+m<1$ we have $|1-\zeta|^{-\sigma-m} \leq 2 \sqrt{2}$. Since $K-m>1$ the integral can be bounded as in the case $\sigma>0$, so that

$$
\begin{aligned}
\left|R S_{K}(\tau)\right| & \leq \frac{2 \sqrt{2}}{\pi^{2}}\left(\frac{1}{\sqrt{2 \pi} a}\right)^{K+1}(5.79)^{m / 2} \Gamma\left(\frac{K+1}{2}\right) \cdot 9.577048(7.49)^{(K-m-1) / 2} \\
& \leq \frac{9.577048 \cdot 2 \sqrt{2}}{7.49 \pi^{2}}\left(\frac{5.79}{7.49}\right)^{m / 2}\left(\frac{\sqrt{7.49}}{\sqrt{2 \pi} a}\right)^{K+1} \Gamma\left(\frac{K+1}{2}\right) \\
& \leq \frac{1}{2}(9 / 10)^{m}\left(\frac{1.1}{a}\right)^{K+1} \Gamma\left(\frac{K+1}{2}\right)
\end{aligned}
$$

finishing the proof of (4.7).

\section{Formulas FOR THE TERMS}

Recall the formula for the coefficients in (3.9):

$$
C_{k}(p)=\frac{e^{-\pi i / 8}}{4(4 \sqrt{\pi})^{k}} \int_{\searrow i p} \frac{e^{-\frac{\pi i}{2}(w-i p)^{2}}}{\cosh \frac{\pi}{2} w} P_{k}(\sqrt{\pi}(w-i p)) d w
$$

with $P_{0}(x)=1$ so that for $k=0$ we get

$$
C_{0}(p)=\frac{e^{-\pi i / 8}}{4} \int_{\searrow i p} \frac{e^{-\frac{\pi i}{2}(w-i p)^{2}}}{\cosh \frac{\pi}{2} w} d w=\frac{e^{\pi i\left(\frac{p^{2}}{2}+\frac{3}{8}\right)}-i \sqrt{2} \cos \frac{\pi}{2} p}{2 \cos \pi p}=: F(p) .
$$

The evaluation of this integral is classic and can be found in Chandrasekharan [3, p. 35].

That the function $F(p)$ is entire follows easily from the integral expression or by noticing that the zeros of $\cos \pi p$ are also zeros of the numerator.

The Hermite polynomials may be defined by the formula

$$
D^{k}\left(e^{-x^{2}}\right)=(-1)^{k} e^{-x^{2}} H_{k}(x) \text {. }
$$

From this it is clear that

$$
D^{k}\left(e^{-\frac{\pi i}{2}(w-i p)^{2}}\right)=e^{-\frac{\pi i}{2}(w-i p)^{2}}\left(\frac{e^{3 \pi i / 4} \sqrt{\pi}}{\sqrt{2}}\right)^{k} H_{k}\left(\frac{e^{\pi i / 4} \sqrt{\pi}}{\sqrt{2}}(w-i p)\right) .
$$

The path of integration in (5.2) depends on $p$. But an easy application of Cauchy's Theorem (recall that $-1<p \leq 1$, and that we modified the path in case $p=1$ as explained in the paragraph following formula (3.5)) allows one to substitute this path for the straight line through 0 with direction $e^{-\pi i / 4}$. Then it is 
easy to justify differentiation (with respect to $p$ ) under the integral sign and restore the old path afterwards. In this way we get

$$
F^{(k)}(p)=\frac{e^{-\pi i / 8}}{4}\left(\frac{e^{3 \pi i / 4} \sqrt{\pi}}{\sqrt{2}}\right)^{k} \int_{\searrow i p} \frac{e^{-\frac{\pi i}{2}(w-i p)^{2}}}{\cosh \frac{\pi}{2} w} H_{k}\left(\frac{e^{\pi i / 4} \sqrt{\pi}}{\sqrt{2}}(w-i p)\right) d w .
$$

From (3.6) and (3.7) it is not difficult to see that the degree of the polynomial $P_{k}(z)$ is $3 k$. Also, $P_{k}(z)$ is even for $k$ even and odd for $k$ odd. Since the Hermite polynomials are a basis of the space of all polynomials and the parity of $H_{k}(x)$ is that of $k$ we can get coefficients $d_{j}^{(k)}$ such that

$$
\begin{aligned}
& \frac{\pi^{2 k} P_{k}(\sqrt{\pi}(w-i p))}{(4 \sqrt{\pi})^{k}} \\
& \quad=\sum_{j=0}^{\lfloor 3 k / 2\rfloor}\left(\frac{\pi}{2 i}\right)^{j} d_{j}^{(k)}\left(\frac{e^{3 \pi i / 4} \sqrt{\pi}}{\sqrt{2}}\right)^{3 k-2 j} H_{3 k-2 j}\left(\frac{e^{\pi i / 4} \sqrt{\pi}}{\sqrt{2}}(w-i p)\right) .
\end{aligned}
$$

This is equivalent to

$$
U_{k}(x):=\left(\frac{e^{3 \pi i / 4}}{\sqrt{2}}\right)^{k} P_{k}\left(x \sqrt{2} e^{-\pi i / 4}\right)=\sum_{j=0}^{\lfloor 3 k / 2\rfloor}(-1)^{k} d_{j}^{(k)} H_{3 k-2 j}(x) .
$$

Combining (5.1), (5.3) and (5.4) we get

$$
C_{k}(p)=\frac{1}{\pi^{2 k}} \sum_{j=0}^{\lfloor 3 k / 2\rfloor}\left(\frac{\pi}{2 i}\right)^{j} d_{j}^{(k)} F^{(3 k-2 j)}(p) .
$$

By (3.7) the polynomials $U_{k}(x)$ satisfy the following relation:

$$
\begin{aligned}
\sum_{k=0}^{\infty} U_{k}(x) \tau^{k}=g\left(\frac{e^{3 \pi i / 4}}{\sqrt{2}} \tau,\right. & \left.x \sqrt{2} e^{-\pi i / 4}\right) \\
= & \exp \left\{-\left(\sigma-\frac{1}{4 \tau^{2}}\right) \log (1-2 x \tau)+\frac{x}{2 \tau}+\frac{x^{2}}{2}\right\}
\end{aligned}
$$

To determine the polynomials $U_{k}(x)$ we differentiate (5.7) logarithmically with respect to $x$ and get

$$
\sum_{k=0}^{\infty} U_{k}^{\prime}(x) \tau^{k}=\frac{2\left(\sigma-x^{2}\right) \tau}{1-2 x \tau} \sum_{k=0}^{\infty} U_{k}(x) \tau^{k}
$$

from which we derive the relation

$$
U_{k}^{\prime}(x)=2\left(\sigma-x^{2}\right) U_{k-1}(x)+2 x U_{k-1}^{\prime}(x) .
$$

Extending the definition of $d_{j}^{(k)}$ so that $d_{j}^{(k)}=0$ for $j<0$ and $j>3 k / 2$, we will have

$$
\begin{aligned}
\sum_{j}(-1)^{k} d_{j}^{(k)} H_{3 k-2 j}^{\prime}(x)=2\left(\sigma-x^{2}\right) & \sum_{j}(-1)^{k-1} d_{j}^{(k-1)} H_{3 k-3-2 j}(x) \\
& +2 x \sum_{j}(-1)^{k-1} d_{j}^{(k-1)} H_{3 k-3-2 j}^{\prime}(x) .
\end{aligned}
$$


It is well known that the Hermite polynomials satisfy

$$
H_{n}^{\prime}(x)=2 n H_{n-1}(x), \quad x H_{n}(x)=\frac{1}{2} H_{n+1}(x)+n H_{n-1}(x)
$$

so that

$$
x^{2} H_{n}(x)=\frac{1}{4} H_{n+2}(x)+\left(n+\frac{1}{2}\right) H_{n}(x)+n(n-1) H_{n-2}(x) .
$$

Therefore, the above relation (5.10) can be written as

$$
\begin{aligned}
\sum_{j} d_{j}^{(k)}(6 k & -4 j) H_{3 k-2 j-1} \\
& =2 \sum_{j} d_{j}^{(k-1)}\left\{\frac{1}{4} H_{3 k-1-2 j}+\left(3 k-3-2 j+\frac{1}{2}\right) H_{3 k-3-2 j}\right. \\
+ & \left.(3 k-3-2 j)(3 k-4-2 j) H_{3 k-5-2 j}\right\}-2 \sigma \sum_{j} d_{j}^{(k-1)} H_{3 k-3-2 j} \\
& -2 \sum_{j} d_{j}^{(k-1)} 2(3 k-3-2 j)\left\{\frac{1}{2} H_{3 k-3-2 j}+(3 k-4-2 j) H_{3 k-5-2 j}\right\} .
\end{aligned}
$$

Separating the coefficients of $H_{3 k-2 j-1}$ and simplifying we arrive at the recurrence

$$
(6 k-4 j) d_{j}^{(k)}=\frac{1}{2} d_{j}^{(k-1)}+(1-2 \sigma) d_{j-1}^{(k-1)}-2(3 k-2 j)(3 k-2 j+1) d_{j-2}^{(k-1)} .
$$

Starting from $d_{0}^{(0)}=1, d_{j}^{(0)}=0$ for $j \neq 0$, these equations determine the $d_{j}^{(k)}$ except those with $3 k=2 j$. To determine these for $k>0$ we can use relation (5.5) for $x=0$ where $P_{k}(0)=0$ :

$$
d_{3 k / 2}^{(k)}=-\sum_{j=0}^{3 k / 2-1}(-1)^{3 k / 2-j} d_{j}^{(k)} \frac{(3 k-2 j) !}{(3 k / 2-j) !}, \quad 3 k \equiv 0 \quad(\bmod 2) .
$$

In order to apply (5.6) we also need to compute $F^{(m)}(p)$. We will use the Taylor expansion of this function. We have

$$
\frac{1}{\cos x}=\sum_{n=0}^{\infty}(-1)^{n} \frac{E_{2 n}}{(2 n) !} x^{2 n}
$$

where, as before, the $E_{n}$ are the Euler numbers.

From this we easily get

$$
F(z)=\frac{e^{\pi i\left(\frac{z^{2}}{2}+\frac{3}{8}\right)}-i \sqrt{2} \cos \frac{\pi}{2} z}{2 \cos \pi z}=\sum_{n=0}^{\infty} c_{2 n} z^{2 n},
$$

where

$$
\begin{aligned}
c_{2 n}=-\frac{i}{\sqrt{2}}\left(\frac{\pi}{2}\right)^{2 n} \sum_{k=0}^{n} \frac{(-1)^{k}}{(2 k) !} 2^{2 n-2 k} & \frac{(-1)^{n-k} E_{2 n-2 k}}{(2 n-2 k) !} \\
& +e^{3 \pi i / 8} \sum_{j=0}^{n}(-1)^{j} \frac{E_{2 j}}{(2 j) !} \frac{i^{n-j} \pi^{n+j}}{(n-j) ! 2^{n-j+1}} .
\end{aligned}
$$

\section{Some useful Bounds}

In this section we will obtain some bounds that will be needed if we want to compute $\mathcal{R}(s)$ with an absolute error less than a given $\varepsilon>0$ by means of (3.11). 
6.1. Bound for $d_{j}^{(k)}$.

Proposition 6.1. We have

$$
\left|d_{j}^{(k)}\right| \leq D_{j}^{(k)}:=A(\sigma) \frac{2^{j}}{B_{1}^{k}}\left(\frac{\Gamma\left(k+\frac{1}{2}\right)}{(3 k-2 j) !}\right)^{1 / 2}
$$

where

$$
A(\sigma):=\left\{\begin{array}{ll}
9^{\sigma} & \text { for } \sigma \geq 0, \\
2^{-\sigma} & \text { for } \sigma<0,
\end{array} \quad B_{1}:= \begin{cases}1 & \text { for } \sigma \geq 0, \\
2 \sqrt{1-\log 2} & \text { for } \sigma<0 .\end{cases}\right.
$$

Proof. From the orthogonality of the Hermite polynomials and (5.5) we get

$$
\int_{-\infty}^{+\infty} U_{k}(x)^{2} e^{-x^{2}} d x=\sqrt{\pi} \sum_{j=0}^{\lfloor 3 k / 2\rfloor} 2^{3 k-2 j}(3 k-2 j) !\left(d_{j}^{(k)}\right)^{2} .
$$

Now we try to get an estimate of the first integral. By Cauchy's Theorem and (5.7) we have

$$
U_{k}(x)=\frac{1}{2 \pi i} \int_{C} \exp \left\{-\left(\sigma-\frac{1}{4 \tau^{2}}\right) \log (1-2 x \tau)+\frac{x}{2 \tau}+\frac{x^{2}}{2}\right\} \frac{d \tau}{\tau^{k+1}} .
$$

Changing variables $\tau=\zeta /(2 x)$ we get

$$
U_{k}(x)=\frac{(2 x)^{k}}{2 \pi i} \int_{C}(1-\zeta)^{-\sigma} e^{-x^{2} f(\zeta)} \frac{d \zeta}{\zeta^{k+1}},
$$

where $C$ denotes a circle with center at $\zeta=0$ and radius $0<r<1$, and $f(\zeta)$ is given by (4.5).

Now observe that, for every fixed $r$ in $(0,1]$, the function $\theta \mapsto-\operatorname{Re} f\left(r e^{i \theta}\right)$ as a function of $\theta \in(0,2 \pi)$ is maximal for $\theta=\pi$ so that

$$
-\operatorname{Re} f\left(r e^{i \theta}\right) \leq \frac{1}{2}-\frac{1}{r}+\frac{\log (1+r)}{r^{2}}=\frac{r}{3}-\frac{r^{2}}{4}+\frac{r^{3}}{5}-\cdots .
$$

(However, actually we only need this inequality for $r=1, r=8 / 9$ and $r=0.883$ 1)

For $\sigma<0$ we get

$$
\left|U_{k}(x)\right| \leq \frac{(2|x|)^{k}}{2 \pi}(1+r)^{-\sigma} e^{-x^{2} f(-r)} \frac{2 \pi}{r^{k}} .
$$

The above inequality implies that

$$
\int_{-\infty}^{+\infty} U_{k}(x)^{2} e^{-x^{2}} d x \leq\left(\frac{2}{r}\right)^{2 k}(1+r)^{-2 \sigma} \frac{\Gamma(1 / 2+k)}{(1+2 f(-r))^{1 / 2+k}} .
$$

The function $r^{2}(1+2 f(-r))$ attains its maximum on the interval $[0,1]$ for $r=1$. So the best inequality we can get in this way is

$$
\int_{-\infty}^{+\infty} U_{k}(x)^{2} e^{-x^{2}} d x \leq 1.28 \cdot 2^{-2 \sigma}\left(\frac{2}{1-\log 2}\right)^{k} \Gamma(1 / 2+k), \quad \sigma<0 .
$$

\footnotetext{
${ }^{1}$ Each of these three cases may also be proved as follows: Defining $u(\theta)=\operatorname{Re} f\left(-e^{i \theta}\right)-$ $\operatorname{Re} f(-1)$, the inequality is equivalent to $u(\theta)>0$ for $0<\theta<\pi$. It is fairly easy to determine $\epsilon>0$ and $\delta>0$ such that $u(\theta)>0$ for $0<\theta<\epsilon$ and $\pi-\delta<\theta<\pi$. It is also fairly easy to determine an $M$ such that $\left|u^{\prime}(\theta)\right| \leq M$ for all theta in $[\varepsilon, \pi-\delta]$. By means of these ingredients, using the Mean Value Theorem, our inequality (for $\epsilon \leq \theta \leq \pi-\delta$ ) may be reduced to a finite set of numerical inequalities (Maximal Slope Principle).
} 
When $\sigma \geq 0$ we get instead

$$
\int_{-\infty}^{+\infty} U_{k}(x)^{2} e^{-x^{2}} d x \leq\left(\frac{2}{r}\right)^{2 k}(1-r)^{-2 \sigma} \frac{\Gamma(1 / 2+k)}{(1+2 f(-r))^{1 / 2+k}} .
$$

We found that for $r=0.883$ we have

$$
r^{2}(1+2 f(-r)) \geq 1 / 2, \quad(1+2 f(-r))^{-1 / 2} \leq 1.25 \quad \text { and } \quad(1-r)^{-1} \leq 8.55
$$

so that

$$
\int_{-\infty}^{+\infty} U_{k}(x)^{2} e^{-x^{2}} d x \leq 1.25 \cdot 9^{2 \sigma} 2^{3 k} \Gamma(1 / 2+k), \quad \sigma \geq 0 .
$$

From (6.3), (6.5) and (6.6) we easily get (6.1).

6.2. Bounds for $F^{(n)}(p)$. We also need to compute $F^{(n)}(p)$ for $-1 \leq p \leq 1$, to a given precision $\varepsilon$, say. The function $F$ is given explicitly by (5.14), but this expression is not suitable to compute $F^{(n)}(p)$. There are various possible practical procedures: use of the Taylor expansion, use of an integral formula, or use of some other expansion, Chebyshev polynomials, for example.

In all cases we will need to know the magnitude of $F^{(n)}(p)$.

Theorem 6.1. For $n \geq 0$ and $-1 \leq p \leq 1$ we have

$$
\begin{aligned}
& \left|F^{(2 n)}(p)\right| \leq \frac{(2 n) !}{2^{n+1} n !} \pi^{n}, \quad\left|F^{(2 n+1)}(p)\right| \leq 2^{n} \pi^{n} n ! \\
& \quad \text { so that }\left|F^{(k)}(p)\right| \leq F_{k}, \quad \text { where } \quad F_{k}:=(2 \pi)^{\frac{k-1}{2}} \Gamma\left(\frac{k+1}{2}\right) \text { for all } k \geq 0 .
\end{aligned}
$$

In particular, the coefficients of the power series of $F(z)$ satisfy

$$
\left|c_{2 k}^{(0)}\right| \leq \frac{\pi^{k}}{2^{k+1} k !} .
$$

Proof. Gabcke 4, V, Theorem 3, formula (1), p. 109] proves the formula

$$
\begin{aligned}
& \frac{\cos \pi\left(\frac{z^{2}}{2}+\frac{3}{8}\right)}{\cos \pi z}-i \frac{\sin \pi\left(\frac{z^{2}}{2}+\frac{3}{8}\right)-\sqrt{2} \cos \frac{\pi}{2} z}{\cos \pi z} \\
&=\sqrt{2} e^{\pi i / 8} \int_{0}^{\infty} e^{-\pi v^{2} / 2} \frac{\cosh \left(\pi z e^{-\pi i / 4} v\right)}{\cosh \left(\pi e^{-\pi i / 4} v\right)} d v .
\end{aligned}
$$

Assuming $z$ real and taking the complex conjugate we get

$$
2 F(z)=\sqrt{2} e^{-\pi i / 8} \int_{0}^{\infty} e^{-\pi v^{2} / 2} \frac{\cosh \left(\pi z e^{\pi i / 4} v\right)}{\cosh \left(\pi e^{\pi i / 4} v\right)} d v .
$$

Since the two members are entire functions the above equality is true for all $z \in \mathbf{C}$.

Differentiating we get

$$
2 F^{(2 n)}(z)=\sqrt{2} e^{-\pi i / 8} \int_{0}^{\infty} e^{-\pi v^{2} / 2}\left(\pi e^{\pi i / 4} v\right)^{2 n} \frac{\cosh \left(\pi z e^{\pi i / 4} v\right)}{\cosh \left(\pi e^{\pi i / 4} v\right)} d v
$$

and

$$
2 F^{(2 n+1)}(z)=\sqrt{2} e^{-\pi i / 8} \int_{0}^{\infty} e^{-\pi v^{2} / 2}\left(\pi e^{\pi i / 4} v\right)^{2 n+1} \frac{\sinh \left(\pi z e^{\pi i / 4} v\right)}{\cosh \left(\pi e^{\pi i / 4} v\right)} d v .
$$


Now we can apply the bounds of Gabcke $[\mathrm{V}, \S 4$, Theorem 1, p. 139] and get for $-1<z<1$,

$$
2\left|F^{(2 n)}(z)\right| \leq \sqrt{2} \int_{0}^{\infty} e^{-\pi v^{2} / 2}(\pi v)^{2 n} d v=\frac{(2 \pi)^{n}}{\sqrt{\pi}} \Gamma\left(n+\frac{1}{2}\right)
$$

and

$$
2\left|F^{(2 n+1)}(z)\right| \leq \sqrt{2} \int_{0}^{\infty} e^{-\pi v^{2} / 2}(\pi v)^{2 n+1} \sqrt{2} d v=2^{n+1} \pi^{n} \Gamma(n+1) .
$$

We can now obtain a bound for the coefficients $C_{k}(p)$ in the Riemann-Siegel formula (2.4).

Proposition 6.2. For $0 \leq j \leq 3 k / 2$ we have

$$
\left|\frac{1}{\pi^{2 k}}\left(\frac{\pi}{2 i}\right)^{j} d_{j}^{(k)} F^{(3 k-2 j)}(p)\right| \leq T_{k}:=A(\sigma) \frac{\Gamma\left(k+\frac{1}{2}\right)^{1 / 2}}{B_{2}^{k}}
$$

with $A(\sigma)$ defined by (6.2) and

$$
B_{2}:= \begin{cases}\sqrt{\pi}, & \sigma \geq 0 \\ 2 \sqrt{\pi(1-\log 2)}, & \sigma<0 .\end{cases}
$$

Proof. By Theorem 6.1 we have $\left|F^{(m)}(p)\right| \leq(2 \pi)^{(m-1) / 2} \Gamma\left(\frac{m+1}{2}\right)$. Assuming that $\sigma \geq 0$ we get by (6.1),

$$
\begin{aligned}
T:=\mid \frac{1}{\pi^{2 k}}\left(\frac{\pi}{2 i}\right)^{j} d_{j}^{(k)} & F^{(3 k-2 j)}(p) \mid \\
& \leq \frac{1}{\pi^{2 k}} \frac{\pi^{j}}{2^{j}} 9^{\sigma} 2^{j}\left(\frac{\Gamma\left(k+\frac{1}{2}\right)}{(3 k-2 j) !}\right)^{1 / 2}(2 \pi)^{\frac{3 k-2 j-1}{2}} \Gamma\left(\frac{3 k-2 j+1}{2}\right) .
\end{aligned}
$$

Simplifying, we obtain

$$
T \leq \frac{9^{\sigma}}{\sqrt{2 \pi}} \frac{1}{2^{j}}\left(\frac{2^{3 / 2}}{\pi^{1 / 2}}\right)^{k}\left(\frac{\Gamma\left(k+\frac{1}{2}\right)}{(3 k-2 j) !}\right)^{1 / 2} \Gamma\left(\frac{3 k-2 j+1}{2}\right) .
$$

If we call this bound $A_{j}$ we get

$$
\frac{A_{j+1}}{A_{j}}=\left(\frac{3 k-2 j}{3 k-2 j-1}\right)^{1 / 2}>1 .
$$

It follows that $A_{j} \leq A_{\lfloor 3 k / 2\rfloor}$, so that

$$
T \leq \frac{9^{\sigma}}{\sqrt{2 \pi}} \frac{2^{3 k / 2}}{\pi^{k / 2}} \frac{1}{2^{\lfloor 3 k / 2\rfloor}} \Gamma\left(k+\frac{1}{2}\right)^{1 / 2} \sqrt{\pi} \leq 9^{\sigma} \frac{\Gamma\left(k+\frac{1}{2}\right)^{1 / 2}}{\pi^{k / 2}}, \quad \sigma \geq 0 .
$$

For $\sigma<0$ an analogous reasoning gives the bound

$$
T \leq 2^{-\sigma} \frac{\Gamma\left(k+\frac{1}{2}\right)^{1 / 2}}{(2 \sqrt{1-\log 2} \sqrt{\pi})^{k}}, \quad \sigma<0 .
$$

\section{ACKNOWLEDGEMENT}

I am thankful to Dr. J. van de Lune (Hallum, The Netherlands, formerly at CWI, Amsterdam) for his linguistic assistance when writing this paper and for his interest in the topic. 


\section{REFERENCES}

1. J. Arias de Reyna, High precision computation of Riemann's Zeta function by the RiemannSiegel formula, II (to appear).

2. M. V. Berry, The Riemann-Siegel expansion for the zeta function: High orders and remainders, Proc. R. Soc. Lond. A 450 (1995), 439-462. MR1349513 (96f:11105)

3. K. Chandrasekharan, Introduction to Analytic Number Theory, Springer-Verlag, Berlin, 1968. MR 0249348 (40:2593)

4. W. Gabcke, Neue Herleitung und explizite Restabschätzung der Riemann-Siegel Formel. Dissertation zur Erlangung des Doktorgrades der Mathematisch-Naturwissenschaftlichen Fakultät der Georg-August-Universität zu Göttingen, Göttingen, 1979.

5. W. F. Galway, Analytic computation of the prime counting function, Dissertation, Urbana,

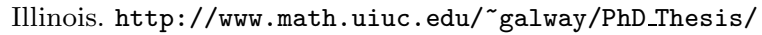

6. X. Gourdon, The $10^{13}$ first zeros of the Riemann Zeta function, and zeros computation at very large height. http://numbers. computation.free.fr/Constants/Miscellaneous/ zetazeros1e13-1e24.pdf

7. B. Riemann, Über die Anzahl der Primzahlen unter einer gegebenen Grösse, Monatsber. Akad. Berlin, 1859, 671-680. (Also in Riemann's Gesammelte Werke 8].)

8. B. Riemann, Bernhard Riemann. Gesammelte Mathematische Werke, Wissenschafticher Nachlass und Nachträge, Based on the edition by Heinrich Weber and Richard Dedekind. Edited and with a preface by Raghavan Narasimhan. BSB B. G. Teubner Verlagsgesellschaft, Leipzig, Springer-Verlag, Berlin, 1990. MR1066697 (91j:01070a)

9. C. L. Siegel, Uber Riemann's Nachlaß zur analytischen Zahlentheorie, Quellen und Studien zur Geschichte der Mathematik, Astronomie und Physik 2 (1932), 45-80. Reprinted in [10, $1,275-310$.

10. C. L. Siegel, Carl Ludwig Siegel's Gesammelte Abhandlungen (edited by K. Chandrasekharan and H. Maaß), Springer-Verlag, Berlin, 1966. MR0197270 (33:5441)

11. E. C. Titchmarsh, The zeros of the Riemann zeta-function, Proc. Roy. Soc. London, 151 (1935), 234-255, and 157 (1936), 261-263.

Facultad de Matemáticas, Universidad de Sevilla, Apdo. 1160, 41080-Sevilla, Spain

E-mail address: arias@us.es 